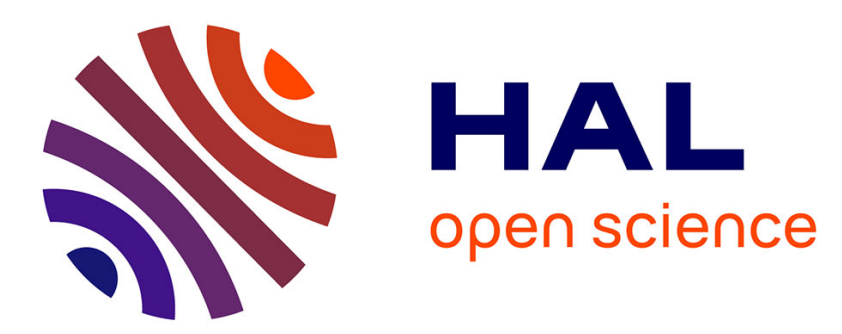

\title{
High efficient computational integral imaging reconstruction based on parallel-group projection (PGP) method
}

Shasha Shi, Patrick Gioia, Gérard Madec

\section{- To cite this version:}

Shasha Shi, Patrick Gioia, Gérard Madec. High efficient computational integral imaging reconstruction based on parallel-group projection (PGP) method. 2nd Annual SMPTE: International Conference on Stereoscopic 3D for Entertainment \& Media, Jun 2011, New York, United States. 10.5594/M001429 . hal-02301746

\section{HAL Id: hal-02301746 \\ https://hal.science/hal-02301746}

Submitted on 30 Sep 2019

HAL is a multi-disciplinary open access archive for the deposit and dissemination of scientific research documents, whether they are published or not. The documents may come from teaching and research institutions in France or abroad, or from public or private research centers.
L'archive ouverte pluridisciplinaire HAL, est destinée au dépôt et à la diffusion de documents scientifiques de niveau recherche, publiés ou non, émanant des établissements d'enseignement et de recherche français ou étrangers, des laboratoires publics ou privés. 
Author(s)

\begin{tabular}{|c|c|c|c|c|c|}
\hline First Name & $\begin{array}{l}\text { Middle } \\
\text { Name }\end{array}$ & Surname & Role & Email & SMPTE Member? \\
\hline Shasha & & Shi & $\begin{array}{l}\text { Presenter } \\
\text { Author }\end{array}$ & $\begin{array}{l}\text { Shasha.shi@telecom- } \\
\underline{\text { bretagne.eu }}\end{array}$ & no \\
\hline Patrick & & Gioia & Author & $\begin{array}{l}\text { Patrick.gioia@orange- } \\
\text { ttgroup.com }\end{array}$ & no \\
\hline Gérard & & Madec & Author & $\begin{array}{l}\text { Gerard.madec@telec } \\
\text { om-bretagne.eu }\end{array}$ & no \\
\hline
\end{tabular}

Affiliation

\begin{tabular}{|l|l|l|}
\hline Organization & Address & Country \\
\hline Orange Labs & $\begin{array}{l}\text { 4 Rue Clos Courtel } \\
35510 \text { CESSON SEVIGNE }\end{array}$ & France \\
\hline Télécom-Bretagne & $\begin{array}{l}\text { Technopôle Brest-Iroise CS-83238 } \\
\text { 29238 BREST }\end{array}$ & France \\
\hline
\end{tabular}

Author(s) - repeat Author and Affiliation boxes as needed--

\begin{tabular}{|l|l|l|l|l|}
\hline First Name & Middle Name & Surname & Role & Email \\
\hline Shasha & Shi & $\begin{array}{l}\text { Presenter } \\
\text { Author }\end{array}$ & $\begin{array}{l}\frac{\text { Shasha.shi@t }}{\text { elecom- }} \\
\text { bretagne.eu }\end{array}$ \\
\hline Patrick & & Gioia & Author & $\begin{array}{l}\frac{\text { Patrick.gioia@ }}{\text { orange- }} \\
\text { ttgroup.com }\end{array}$ \\
\hline Gérard & Madec & Author & $\begin{array}{l}\frac{\text { gerard.madec }}{\text { @telecom- }} \\
\text { bretagne.eu }\end{array}$ \\
\hline
\end{tabular}

Affiliation

Organization

Address

Country

The authors are solely responsible for the content of this technical presentation. The technical presentation does not necessarily reflect the official position of the Society of Motion Picture and Television Engineers (SMPTE), and its printing and distribution does not constitute an endorsement of views which may be expressed. This technical presentation is subject to a formal peer-review process by the SMPTE Board of Editors, upon completion of the conference. Citation of this work should state that it is a SMPTE meeting paper. EXAMPLE: Author's Last Name, Initials. 2010. Title of Presentation, Meeting name and location: SMPTE. For information about securing permission to reprint or reproduce a technical presentation, please contact SMPTE at jwelch@smpte.org or 914-761-1100 (3 Barker Ave., White Plains, NY 10601). 


\begin{tabular}{|l|l|l|}
\hline Orange Labs & $\begin{array}{l}\text { 4 Rue Clos Courtel } \\
35510 \text { CESSON SEVIGNE }\end{array}$ & France \\
\hline Télécom-Bretagne & $\begin{array}{l}\text { Technopôle Brest-Iroise CS-83238 } \\
29238 \text { BREST }\end{array}$ & France \\
\hline
\end{tabular}

Publication Information

\begin{tabular}{|l|l|}
\hline Pub ID & Pub Date \\
\hline 2010 Fall Conference Paper 1569426135 & \\
\hline
\end{tabular}

SMPTE Meeting Presentation

\section{High efficient Computational Integral Imaging Reconstruction based on Parallel-Group Projection (PGP) Method}

\section{Shasha Shi, PHD Student}

Orange Labs, Télécom-Bretagne, 4 Rue Clos Courtel 35510 CESSON SEVIGNE France, shasha.shi@telecom-bretange.eu

\section{Patrick Gioia, Research engineer}

Orange Labs, 4 Rue Clos Courtel 35510 CESSON SEVIGNE France, patrick.gioia@orangeftgroup.com

\section{Gérard Madec, Research teacher}

Télécom-Bretagne, Technopôle Brest-Iroise CS-83238 29238 BREST France, gerard.madec@telecom-bretagne.eu

\section{Written for presentation at the}

2nd Annual SMPTE International Conference on Stereoscopic 3D for Entertainment \& Media

\footnotetext{
The authors are solely responsible for the content of this technical presentation. The technical presentation does not necessarily reflect the official position of the Society of Motion Picture and Television Engineers (SMPTE), and its printing and distribution does not constitute an endorsement of views which may be expressed. This technical presentation is subject to a formal peer-review process by the SMPTE Board of Editors, upon completion of the conference. Citation of this work should state that it is a SMPTE meeting paper. EXAMPLE: Author's Last Name, Initials. 2010. Title of Presentation, Meeting name and location: SMPTE. For information about securing permission to reprint or reproduce a technical presentation, please contact SMPTE at jwelch@smpte.org or 914-761-1100 (3 Barker Ave., White Plains, NY 10601).
} 
Abstract. Integral imaging is an auto-stereoscopic 3D technique presenting remarkable advantages over classical stereo. In this paper we propose a new computational integral imaging reconstruction (CIIR) technique based on Parallel-Group Projection (PGP) method to improve the performance of CIIR. Different from previous CIIR techniques which project each point of integral image (II) to the reconstructed plane pixel by pixel, the proposed method reconstruct the $3 D$ image by mapping a series of sub image (SI) onto the reconstructed plane successively, where each SI records pixels from parallel light rays with identical viewing angle. According to experimental results, this approach is able to simplify the calculation of reconstruction process and improve the quality of reconstructed $3 D$ image.

Keywords. integral imaging, computational reconstruction, parallel-group projection, sub image 


\section{Introduction}

3D technology has received considerable attention since 1838 when Charles Wheatstone first described his theories on stereopsis. In spite of its significant progress for past decades of years, 3D technology has not yet accomplished its promise of offering natural viewing effect for spectators. Currently provided stereoscopic or auto-stereoscopic systems produce relief perception to viewers by simulating binocular disparity between two eyes. In these systems, slightly different images are sent to left eye and right eye separately to construct a 3D perception for viewers. Due to its relative simplicity, this kind of 3D system is widely utilized for today's 3D products but it contains some inevitable defects such as the discontinuous viewpoint for different viewing directions and irrespective effect between accommodation and convergence operations [1].

According to the inborn limitations of conventional stereoscopic and auto-stereoscopic systems, next generation 3D systems providing parallax in all directions, make accommodation and convergence work in unison, are eagerly required. Integral imaging is proposed as a practical and prospective technique for this purpose.

A typical integral imaging system could be dived into two stages, named as acquisition and display. Figure 1-1 illustrates the optical working principle for the integral imaging system. In the pickup process, the direction and intensity information of light rays come from 3D object are captured through an array of convex lenses or pinholes and then recorded by a 2D image sensor, as shown in the left picture of Figure 1-1. The recorded image through each lens or pinhole is so called elemental image (EI), which represent the 3D objet from a specific

perspective. An integral image (II) is composed of many Els, with their number corresponding to the number of lenses or pinholes. While in the display side, the decoded array of elemental images is exposed behind the same optical devices (lenses or pinholes array) to reconstruct the light rays of the 3D objet in the reverse direction, shown in the right picture of Figure 1-1. For optical integral imaging reconstruction (OIIR) operation, the diffraction and restrict of optical devices introduced the quality degradation and the viewing angle limitation of reconstructed image. To overcome these problems, computational integral imaging reconstruction (CIIR) method has been developed recently [2, 6 and 7], the CIIR is achieved by simulating the projection effect of optical rays of Els in OIIR using a computer built virtual pinhole. In this method, the reconstructed output image (ROI) could be generated at any arbitrary distance from virtual pinhole array without device degradation and optical diffraction effect.

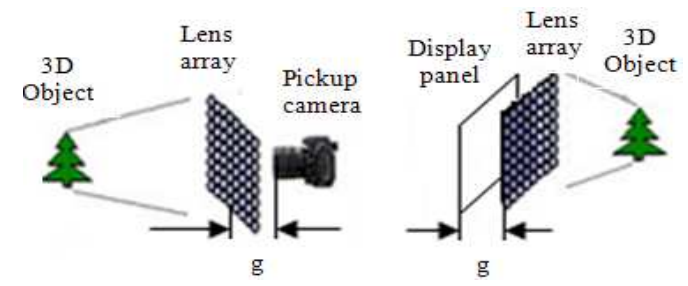

Figure 1-1 Optical setup for generating (left) and displaying (right) 3D scene in integral imaging system

Although computational integral imaging system contains several useful superiorities, there still exits some limitations which obstruct its practical utilization, such as low ROI quality and high calculation complexity. In this paper, a new CIIR approach based on parallel-group projection (PGP) method was proposed to solve these problems.

This paper is organized as follows. Section 2 presents some related works on computational integral imaging reconstruction. The proposed CIIR method based on Parallel-Group Projection 
is explained in section 3. Simulation results and detailed analysis are reported in section 4. Finally, conclusions and discussion are drawn in section 5.

\section{Related work}

Figure 2-1 illustrates the process of conventional CIIR method. In this method, each elemental image is inversely projected through its corresponding pinhole, magnified by the magnification factor $\mathrm{M}$, which is the quotient between the distance from the virtual pinhole array to the reconstructed image plane (denoted by ' $z$ ' in figure 2-1) and the distance from elemental image plane to pinhole array (denoted by ' $g$ ' in figure 2-1). The magnified pixels are overlapped by each other in reconstructed image plane to reproduce the ROI in the determined distance [2]. Series of reconstructed image with different ' $z$ ' value are generated continuously to compose a volumetric reconstruction of the $3 \mathrm{D}$ scene. This CIIR approach is straightforward but it contains a crucial problem about image quality degradation. This quality degradation comes from the magnification process, where each pixel is enlarge and superposed by another pixel, and the superimposition between adjacent pixels causes the undesirable interference result in output image.

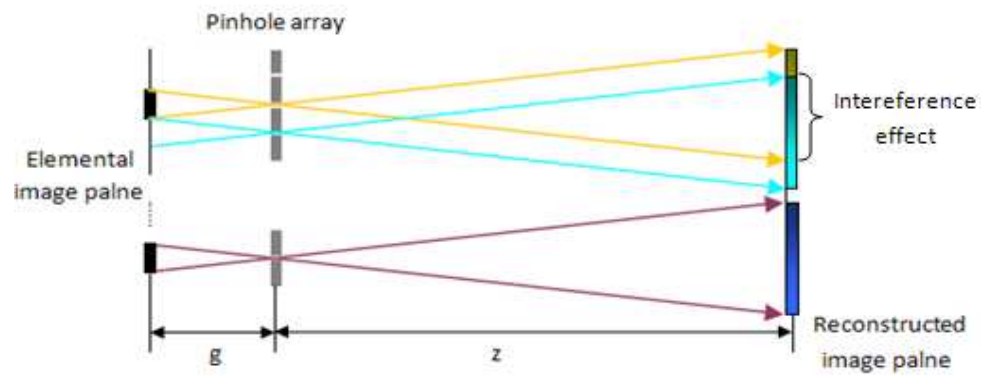

Figure 2-1 Reconstruction principle of traditional CIIR method

To solve this quality degradation problem, another CIIR method using pixel to pixel mapping technique was proposed in reference [5], the principle of this approach is described in figure 22. It removes the magnification process of previous CIIR techniques, generates a dot-pattern $\mathrm{ROI}$ by projecting each pixel of the elemental images into output plane, and then fulfills the empty pixels in this dot-pattern image by the linear interpolation method. Because of the exact generation of pixel position, this method can remove the interference effect betweens adjacent pixels and improve the quality of reconstructed image. However, due to the repetitive projecting of each pixel in elemental images, the calculation complexity is still very important. Moreover, as a result of the separated pixel projection process, this method induced an undesirable dotdiscrete effect for ROI.

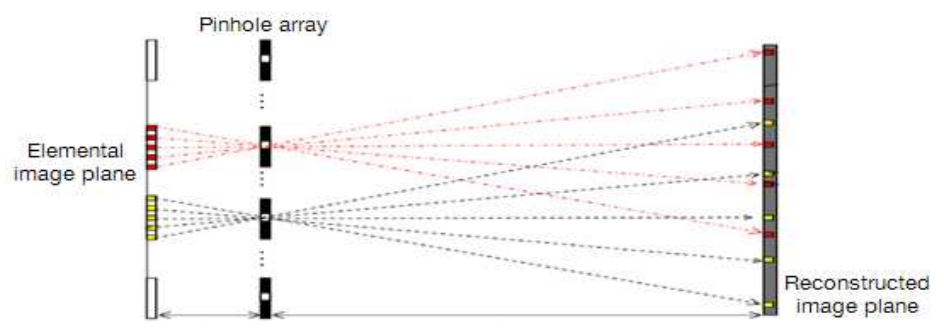

Figure 2-2 Reconstruction principle of pixel-to-pixel mapping CIIR method

To overcome all the inconvenient of previous techniques, we proposed a new CIIR approach using Parallel-Group Projection (PGP) method in this paper. Different from exited techniques, this new approach propagates sub image (SI) instead of original elemental images through the 
computer synthesized pinhole array to reconstruct the ROI, where $\mathrm{SI}$ is a special form of 2D image which records the information of parallel light rays from the given 3D scene [8], so it is nature that the reverse mapping rays of each pixel from the SI into the reconstructed image are also mutually parallel. Therefore, we considered reconstructing the ROI by parallel-group projection instead of pixel-to-pixel projection to exploit this mapping regularity. This new CIIR technique is able to eliminate the interference and dot-discrete effect in conventional generated $\mathrm{ROI}$ and increase the efficiency of previous CIIR methods significantly.

\section{Process of Proposed CIIR Method}

A typical scheme of integral imaging system using the proposed CIIR method is illustrated in Figure 3-1. This system can be achieved by three steps. Firstly, sub images recording the light information of parallel rays are generated by OpenSceneGraph (OSG) 3D graphics toolkit using special configured cameras [3]. Thereafter, the obtained SIs are encoded and decoded by proper compression technique. Finally, in the display side the output 3D image is reconstructed by proposed CIIR technique.

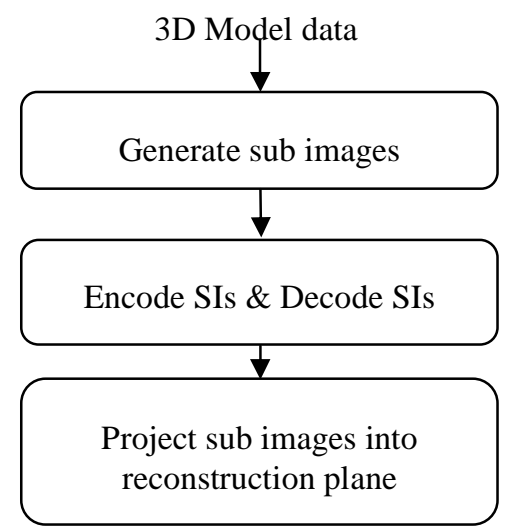

Figure 3-1 Block diagram of proposed integral imaging system

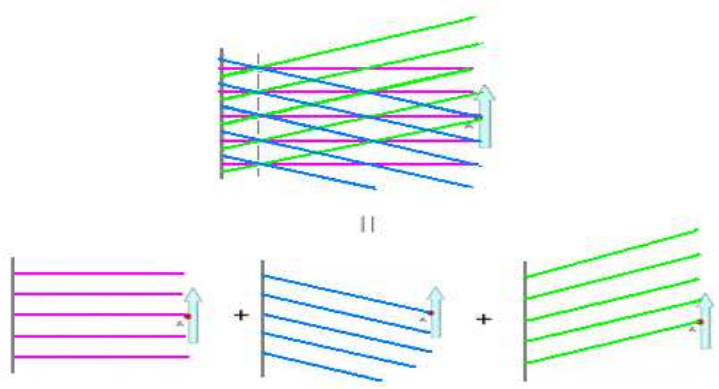

Figure 3-2 Concept of proposed CIIR method

In the following part, we will concern on the concrete procedure of proposed CIIR. As figure 3-2 illustrated, the projected image of the original Els through a computer synthesized pinhole array could be represented as the accumulation of the mapped result of each SI through the same virtual pinhole array. Due to the known parallel character of the mapping light rays [4], all the mapped pixels from one SI are arranged as a grid with a given interval in the reconstructed plane. Therefore, from the location of one projected pixel we are able to estimate the projected coordinate of every pixel by simply displacing the first mapped pixel with an specific offset, and the eventually ROI is formed by accumulating the projected result of every SI on the reconstructed plane. 
Figure 3-3 illustrates the detailed calculation process of the proposed CIIR method, which is carried out by three steps. In the beginning, we project the first pixel of a SI into the reconstructed image plane through the computer synthesized pinhole array based on the geometric optical diagram shown in figure 3-4. According to this diagram, the projected coordinate of one pixel from SI could be calculated by the following equation:

$$
\begin{array}{r}
\left(X_{0}, Y_{0}\right)=(-x * M,-y * M) \\
M=(Z * \tan \theta * 2) /((n-1) * \emptyset)
\end{array}
$$

Where ' $\mathrm{X}_{0}$ ', ' $\mathrm{Y}_{0}$ ' express the mapped pixel coordinates in ROI. ' $x$ ' and ' $y$ ' represent the index of the projecting $S I$, which are ranged as $(0, \pm 1, \pm 2 \ldots \pm(n-1) / 2)$. ' $n$ ' is the number of $S I$ in horizontal or vertical axis. ' $M$ ' is the magnification factor. ' $\varnothing$ ' is the size of pixel in SI. ' $\theta$ ' is the viewing angle of the marginal SI

Once we get the correct projected position of the first pixel in each SI from formula (1) and (2), the second step is to deduce the mapped coordinates of the other pixels in this SI through the regular layout of projected pixels, which could be represented simply by the fowling equation:

$$
(X, Y)=\left(p+X_{0}, q+Y_{0}\right)
$$

Where ' $p$ ' and ' $q$ ' are the position of each pixel in the SI

Finally, the projected outcomes of each sub image are superimposed to each other for constructing the displayed ROI at the determined view distance, and a full 3D volume scene is generated by iteratively repeating this process for variant visual distance.

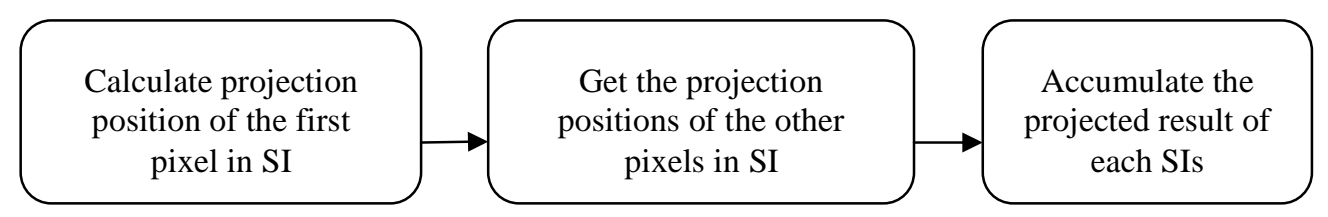

Figure 3-3 Block diagram of proposed CIIR method

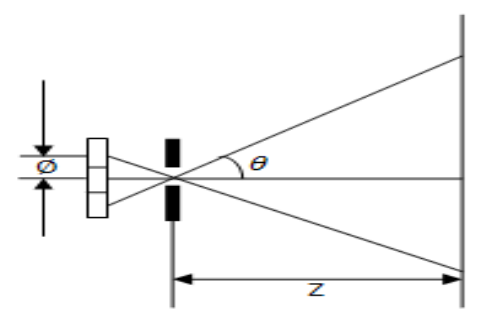

Figure 3-4 Computational reconstruction

Besides its high computational efficiency derived from parallel-group-projection, this CIIR approach contains several other merits compare to previous CIIR methods. First of all, because of its direct application of rendered SI, the transformation and reverse transformation process between II and SIs are no longer necessary in the novel system. Another supplementary advantage lies in the variability of the reconstructed image size, which is in direct proportion to the size of SI, and the size of SI could be adjusted easily by exist interpolation techniques.

\section{Experiments and Results}

The experiment was carried out to demonstrate the effectiveness of proposed approach. In the beginning of the experiment, nine sub images with distinguish viewing angles are rendered by OSG toolkit with orthotropic projective cameras, which are illustrated in figure 4-1. The resolution of each $\mathrm{SI}$ is $512 \times 512$, viewing distance of the virtual camera is 15 , number of $\mathrm{SI}$ in 
horizontal or vertical axis is 3 , and the viewing angle of marginal $\mathrm{SI}$ is $15^{\circ}$. According to the known parameters and formula (2), we can get the magnification factor M equal to 25.
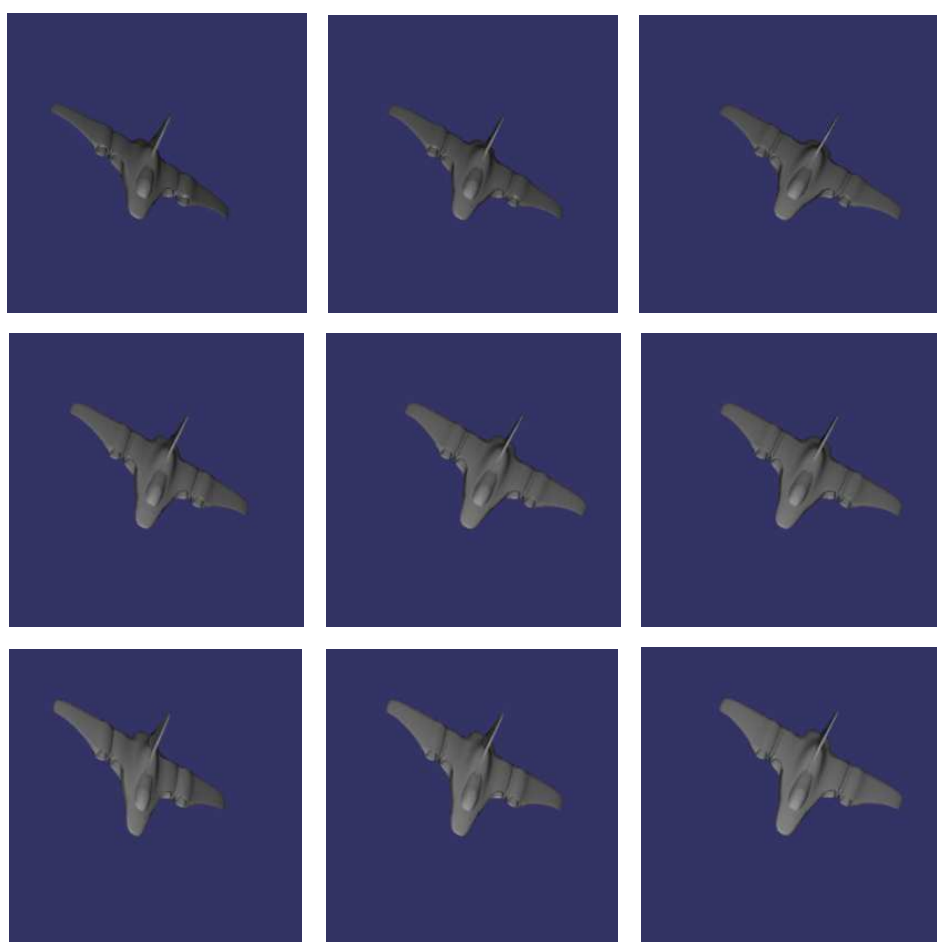

Figure 4-1 Generated SIs for a 3D scene

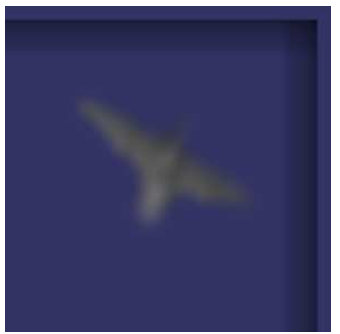

(a)
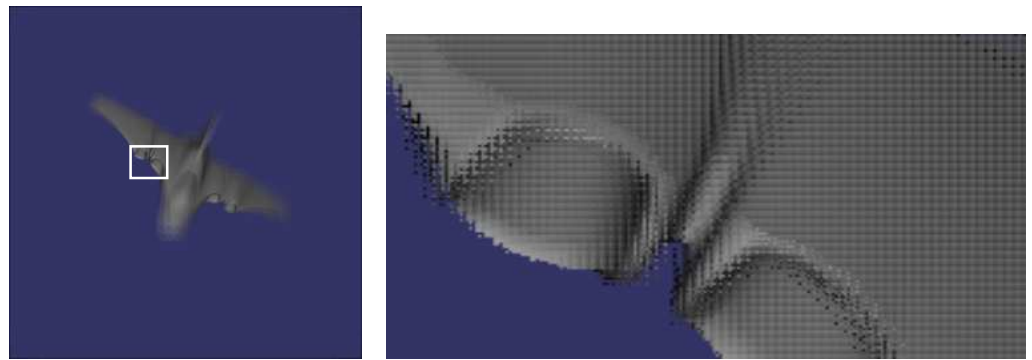

(b) 

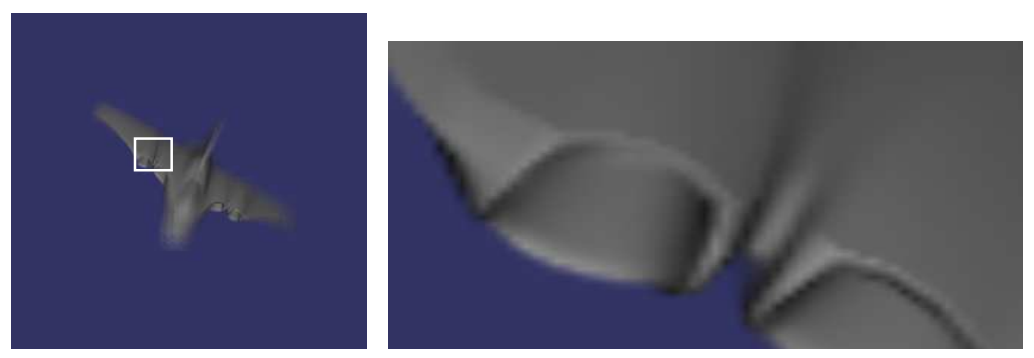

(c)

Figure 4-2 Comparison of reproduced $\mathrm{ROI}$ of three different methods. (a) ROI in conventional method with interference effect. (b) ROI in pixel-to-pixel mapping method with discrete-point effect. (c) ROI in proposed method with higher visual quality

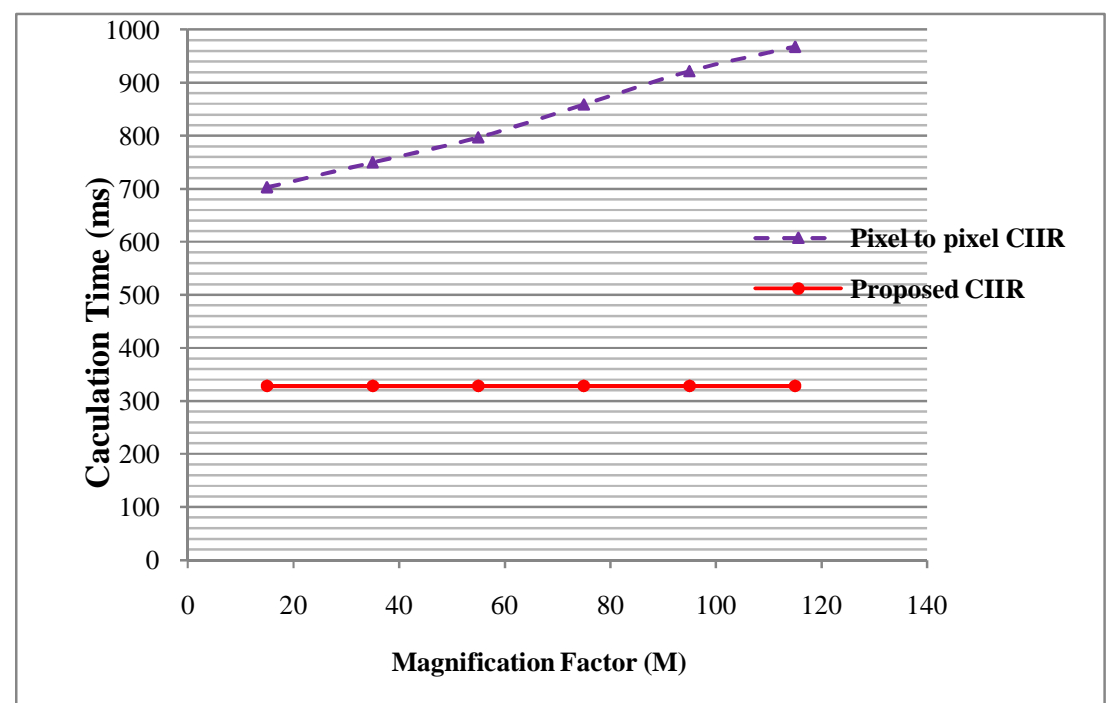

Figure 4-3 Computation cost of pixel-to-pixel and parallel-group projection CIIR

Table 4-1 Comparison of the computation cost of three CIIR methods

\begin{tabular}{llll}
$\begin{array}{l}\text { Magnification } \\
\text { parameter }\end{array}$ & Traditional CIIR & $\begin{array}{l}\text { CIIR using pixel-to- } \\
\text { pixel mapping }\end{array}$ & $\begin{array}{l}\text { CIIR using parallel } \\
\text { group projection }\end{array}$ \\
\hline 15 & 67078 & 703 & 328 \\
\hline 35 & 363218 & 703 & 328 \\
\hline 55 & 897578 & 797 & 328 \\
\hline 75 & 1688047 & 859 & 328 \\
\hline 95 & 2724593 & 922 & 328 \\
\hline 115 & 4004547 & 968 & 328 \\
\hline
\end{tabular}

Three different reconstructed images are generated separately by traditional, pixel-to-pixel based and parallel-group projection based CIIR methods. The performance of CIIR technique is evaluated in terms of image quality and calculation efficiency. Figure 4-2 shows the reproduced $\mathrm{ROI}$ of three techniques with identical parameters. Compare to traditional methods, proposed CIIR approach provide a high quality reconstructed 3D image without interference effect in traditional method and point discrete effect in pixel-to-pixel mapping method.

Table 4-1 and figure 4-3 illustrate the computational cost of the three methods in function of the magnification factor M. According to the exposed data, we can detect that for a reconstructing 
process with a certain magnification factor, the proposed PGP CIIR method shows a significant efficiency progress compare to traditional and pixel-to-pixel mapping method, and its calculation time is not affect by the increasing of magnification factor $M$.

\section{Conclusions}

In this paper we propose a novel CIIR technique based on parallel-group projection method to improve the efficiency and performance of traditional CIIR methods. In this approach, the reconstructed output image is generated by using the parallel-group projection technique to each sub image. Where sub image is an alternative format of $2 \mathrm{D}$ image which recodes the information of parallel light rays from the 3D scene. Exactly and completely pixel position calculation process could avoid the interference problem and the dot-pattern effect of previous CIIR techniques, and the direct projection of SI and the simple mapping regularity of each SI reduce the consuming time of earlier approaches. The experimental results show that our proposed method outperforms the previous CIIR method both in terms of visual quality and in terms of calculation efficiency. In the future, we intend to extend this reconstruction idea from digital visualization system into real optical display system so as to make the present 3D view similar to real life 3D version.

\section{Reference}

[1] Manuel Maritinez-corral, Raul Martinez-Cuenca; Genaro Savedra; Héctor Navarro, Amparo Pons, and Bahram Javidi, "Progresses in 3D Integral Imaging with Optical Process", Journal of Physics: Conference Series 139 (2008).

[2] S Seung-HyunHong, Ju-Seog Jang, and Bahram Javidi, 'Three-dimensional volumetric object reconstruction using computational integral imaging', Optical Society of America, Vol.12, No.3, 9 Rebruary 2004.

[3] Bin-Na-Ra Lee, Yongjoo Cho, Kyoung Shin Park, Sung-Wook Min, Joa-Sang Lim, Min Cheol Whang, and Kang Ryong Park, 'Design and implementation of a Fast Integral Image Rendering Method', ICEC 2006, LNCS 4161,pp.135-140,2006.

[4] D.W.Fellner and C.Hansen, 'Toward the Light Field Display: Autostereoscopic Rendering via a Cluster of Projectors', the Eurographics Association 2006.

[5] Dong-Hak Shin, Hoon Yoo, 'Computational integral imaging reconstruction method of 3D images using pixel-to-pixel mapping and image interpolation', Optics Communications 282 (2009) 2760-2767.

[6] Dong-Choon Hwang, Jae-Sung Park, Kong-Hak Shin, and Eun-Soo Kim, 'Depth-controlled reconstruction of 3D integral image using synthesized intermediate sub-image', Optics Communications 281 (2008) 5991-5997.

[7] Dong-Hak Shin, Byoungho Lee, and Eun-soo Kim, 'Improved Viewing Quality of 3-D Images in Computational Integral Imaging Reconstruction Based on Lenslet Array Model', ETRI journal, Volume 28, Number 4, August 2006.

[8] Jae-Hyeung Park, Joohwan Kim, and Byoungho Lee, 'Three-dimensional Optical Correlation Using a Sub-image Array', Optical Express 5116, 27 June 2005. 